IRSTI 29.19.15

\title{
Features of relaxation processes in methane cryocondensates and weak methane solutions in nitrogen and argon
}

\author{
A.U. Aldiyarov ${ }^{1}$, A.Ye. Nurmukan ${ }^{1}$, D.Yu. Sokolov ${ }^{1}$, \\ A.K. Shinbayeva ${ }^{1 *}$ and M.Zh. Zhubaniyazova ${ }^{2}$ \\ ${ }^{I}$ Institute of Experimental and Theoretical Physics, Al-Farabi Kazakh National University, \\ 71, al-Farabi Ave., 050040, Almaty, Kazakhstan \\ ${ }^{2}$ K. Zhubanov Aktobe Regional State University, A.Moldagulova ave. 34, 030000 Aktobe, Kazakhstan \\ *e-mail:shinbayeva_a@hotmail.com
}

\begin{abstract}
The paper presents results of IR-spectrometric studies of phase transition peculiarities in thin-film cryodeposits of methane and weak solutions of methane in nitrogen and argon in the temperature range from $12 \mathrm{~K}$ to $32 \mathrm{~K}$ and at the deposition pressure of $\mathrm{P}=10^{-4}$ Torr. The relation between the position of the absorption band of deformation vibrations $v_{4}$ and $\alpha-\beta$ phase transition in solid methane is established. Vibrational spectra of cryodeposited mixtures of methane with argon and nitrogen obtained at various ratios of the components demonstrate a correlation between the nitrogen or argon concentration and the absorption band $v 4$ position. The presence of $5 \%$ admixture of argon or nitrogen in the mixture leads to a violation of the reversibility of the $\alpha-\beta$ transition. Although the initial heating of the sample from $\mathrm{T}=16 \mathrm{~K}$ to $\mathrm{T}=26 \mathrm{~K}$ clearly shows transition from the $\beta$-phase to the $\alpha$-phase at $\mathrm{T}=20 \mathrm{~K}$, further heating and thermal cycling in the same temperature range results in the spectrometer signal following a new trajectory, distant from the initial heating curve.
\end{abstract}

Key words: methane, spin-nuclear conversion, low temperature, mixture, argon, nitrogen.

PACS number(s): 63.70.+h, 64.60.-i, 64.70.-p

\section{Introduction}

It is known, that molecules with non-zero spin moments of nuclei can have different nuclear spin states [1-7]. These states are not stable and can be transformed under various external conditions. Usually these processes are very slow due to the weak magnetic interactions between these nuclear spins. Unlike condensed states, the separate existence of each spin state in the gas phase at room temperature is extremely unstable (apart from the hydrogen molecule). The reason is the existence of degenerate levels between the respective spin isomers. These levels are located close the energies of rotational vibrations, resulting in a significant increase in the conversion rate between different spin states. In this respect, the occupancy of each spin state comes into equilibrium with a relatively high rate (considering the temperature). At low temperatures in the condensed state, these degenerate energy states are less involved in the relaxation processes. Thus, different spin states can be obtained and stabilized by ultrafast cooling, when rotational and vibrational relaxations may occur only within the same nuclear spin isomers. As a result, anomalous ratios of absorption intensities in the IR range are observed, which are sometimes referred to as "rotational anomalies" [8]. As such, the respective problem may be formulated as follows: what are the influences of the cryodeposition temperature and the presence of impurities in methane at various concentrations on the nature of the spin-phonon interaction and, as a result, on the phase transition between the $\alpha$ and $\beta$ states of methane cryodeposits?

\section{Experiment and results}

The aim of the study was to investigate the peculiarities of transformations in cryodeposited films of pure methane and its mixtures with argon and nitrogen at different ratios. The results of the IR spectrometric studies of thin-film cryovacuum deposits of methane and weak methane solutions in nitrogen and argon in the temperature range from 12 $\mathrm{K}$ to $32 \mathrm{~K}$, i.e. in the vicinity of the $\alpha-\beta$ transition temperature, are presented. The deposition pressure was $\mathrm{P}_{\mathrm{dep}}=10^{-4}$ Torr, the thickness of the samples varied from 1 to $25 \mu \mathrm{m}$ depending on concentration of the mixture, so that the effective interaction of the globar radiation with methane molecules was the same for different concentrations of the mixture. IR 
measurements were carried out in the vicinity of absorption band of deformation vibrations $v_{4}[9,10]$, which showed high sensitivity to structural transformations of solid methane. To determine the observation frequency $v_{\mathrm{obs}}$, during obtaining thermograms the values near the frequency on a half of absorption amplitude $v_{4}$ in the low-frequency part of the spectrum were selected.

For cryocondensates of pure methane, the interrelation between position of absorption band of deformation vibrations $v_{4}$ with $\alpha-\beta$-phase transition in solid methane is established. Figurel shows the characteristic absorption spectra in frequency range of deformation vibrations of methane cryocondensate thin film formed at $16 \mathrm{~K}(1)$, heated to $30 \mathrm{~K}(2)$ and then cooled to $16 \mathrm{~K}$ (3). The arrows indicate the direction of spectral absorption bands shift with passing the phase transition temperature $\mathrm{T}$ $=20.4 \mathrm{~K}$. As can be seen from the figure, heating of sample from condensation temperature $\mathrm{T}=16 \mathrm{~K}$ to $33 \mathrm{~K}$ leads to a sharp broadening of absorption band $v_{4}$. The positions of the absorption maximum shift to "blue" part of spectrum from $v=1301 \mathrm{~cm}^{-1}$ at $16 \mathrm{~K}$ to $v=1304 \mathrm{~cm}^{-1}$ at $16 \mathrm{~K}$, respectively. At the same time, the absorption half-width varies approximately from $\Delta=13 \mathrm{~cm}^{-1}$ at $16 \mathrm{~K}$ to $\Delta=20 \mathrm{~cm}^{-1}$ at $33 \mathrm{~K}$, respectively.

Reverse temperature decrease of the sample from $33 \mathrm{~K}$ to $16 \mathrm{~K}$ causes the complete recovery of both the $\mathrm{v}_{4}$ band position and the magnitude of absorption amplitude. Based on this fact, we conclude that for thin films of pure methane cryovacuum condensates the $\alpha-\beta$ transition is reversible.

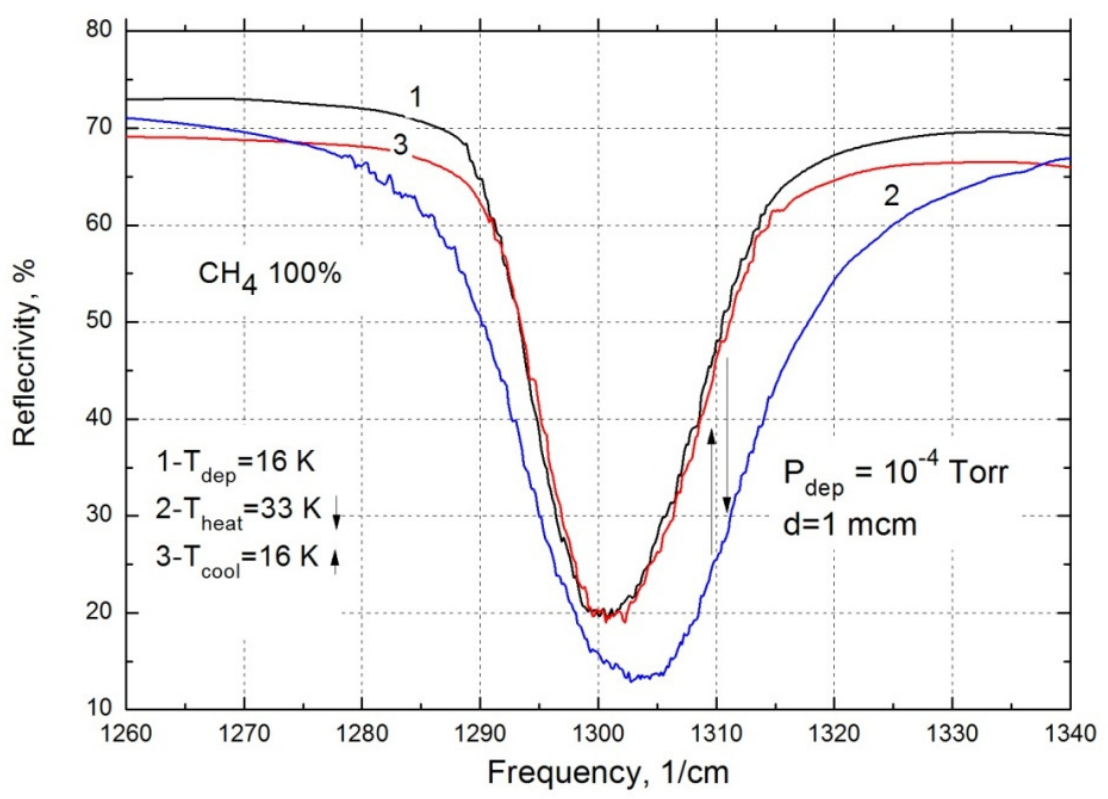

Figure 1 - The absorption spectra of a thin film of cryocondensates of methane formed at $\mathrm{T}=16 \mathrm{~K}(1)$, heated to $\mathrm{T}=30 \mathrm{~K}(2)$ and cooled to $\mathrm{T}=16 \mathrm{~K}$ (3)

To confirm this conclusion and determine the nature of the displacement of the absorption band $v_{4}$ at about the phase transition temperature $\mathrm{T}=20.4 \mathrm{~K}$, measurements of the IR spectrometer signal were conducted at a fixed observation frequency. Figure 2 shows characteristic results of the dependence of the position of the absorption band deformation vibrations of methane molecules (lower graph) on cyclic temperature changes in the substrate (upper graph) in vicinity of the $\alpha-\beta$-transition temperature
$\mathrm{T}=20.4 \mathrm{~K}$. The observation frequency was $v=1310 \mathrm{~cm}^{-1}$, the film thickness was $\mathrm{d}=3 \mu \mathrm{m}$. As can be seen, there is a clear relationship between the direction of change in substrate temperature and the change in the position of the absorption band of deformation vibrations of methane, as well as between the rate of temperature change and the peak shift. The latter is not typical for the first kind phase transitions, which are implemented stepwise in a fairly narrow temperature interval. 


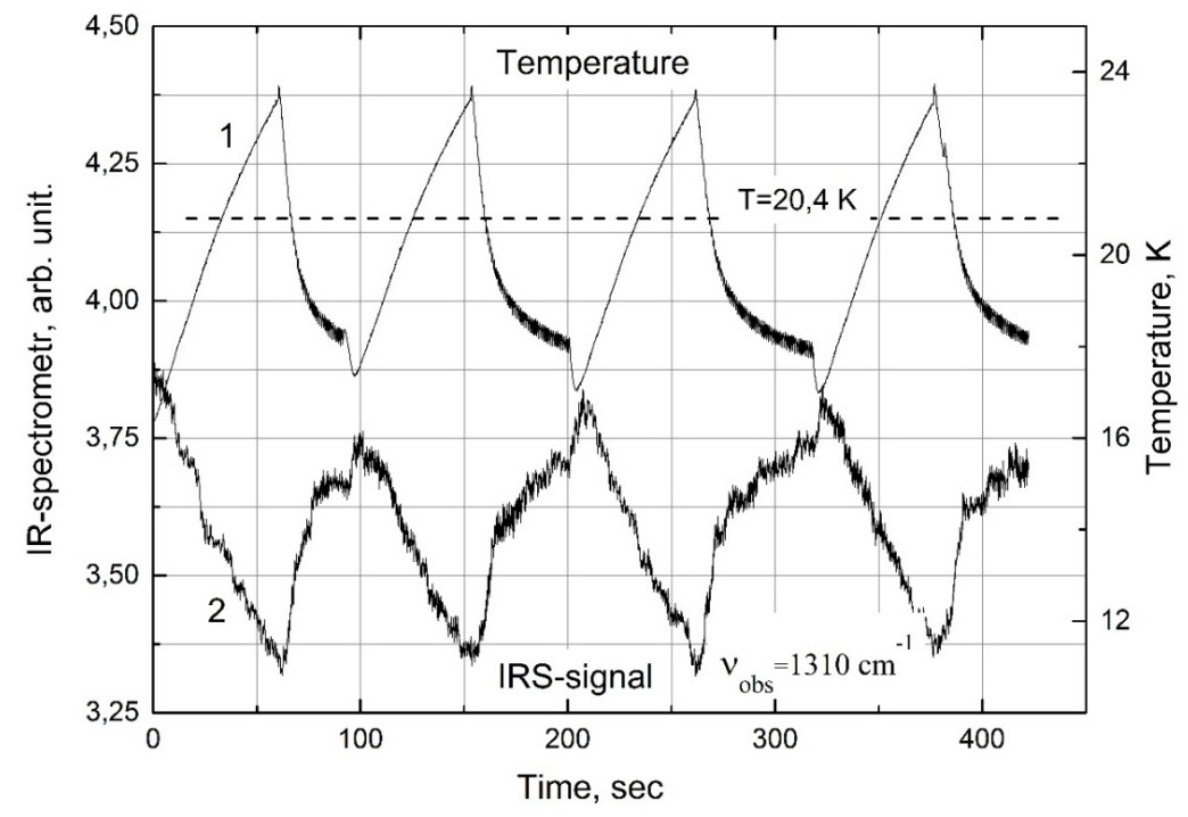

Figure 2 - The character of the change in the position of the absorption band at the observation frequency $v=1310 \mathrm{~cm}^{-1}$ during the cyclic temperature change in the vicinity of $\mathrm{T}=20 \mathrm{~K}$

The observed gradual shift of the absorption band in accordance with the change in temperature is, in our opinion, a manifestation of the spinnuclear conversion processes [2], which take place in the samples of methane cryocondensates, which is actually superimposed on the phase transition. That is, each temperature value corresponds to the equilibrium concentration ratio of the spin-nuclear modifications of methane (meta-ortho-para) and the corresponding position of the absorption band of deformation vibrations. However, due to the dependence of the conversion rate on temperature [3], the time for achieving this equilibrium value should depend on the direction of temperature change. This assumption is also confirmed by the asymmetric behavior of the displacement of the absorption band with respect to the direction of temperature change, which may be due to the influence of the conversion rate on the temperature.

The results of the behavior of weak solid methane solutions with argon and nitrogen in the vicinity of the $\alpha$ - $\beta$-transition temperature of methane are presented below. Concentration ratios were chosen in a way that in one case methane acted as a
$5 \%$ impurity in cryocrystals of nitrogen and argon, and in the other case nitrogen and argon molecules were negligible 5\% impurities in the solid methane structure. Samples were prepared by cocondensation of the gas mixture on a metal substrate at different temperatures. A detailed scheme for carrying out such measurements, the experimental setup and the features of sample preparation are given in our earlier studies $[11,12]$.

Figure 3 shows the vibrational spectra of mixtures of methane with nitrogen (left image) and argon (right image) of the different concentrations in comparison with pure methane. The samples are condensed at $16 \mathrm{~K}$. It can be seen that a decrease in the methane concentration in both cases with argon and nitrogen, leads to a shift in the maximum of the absorption band of deformation vibrations of methane into the "blue" part of the spectrum. Thus, for a mixture with argon, absorption maxima are located at frequencies of $1297 \mathrm{~cm}^{-1}, 1299 \mathrm{~cm}^{-1}, 1301$ $\mathrm{cm}^{-1}$, and mixtures with nitrogen at frequencies of $1297 \mathrm{~cm}^{-1}, 1302 \mathrm{~cm}^{-1}, 1303 \mathrm{~cm}^{-1}$ at methane concentrations of 100,95 and 5 percent, respectively. 

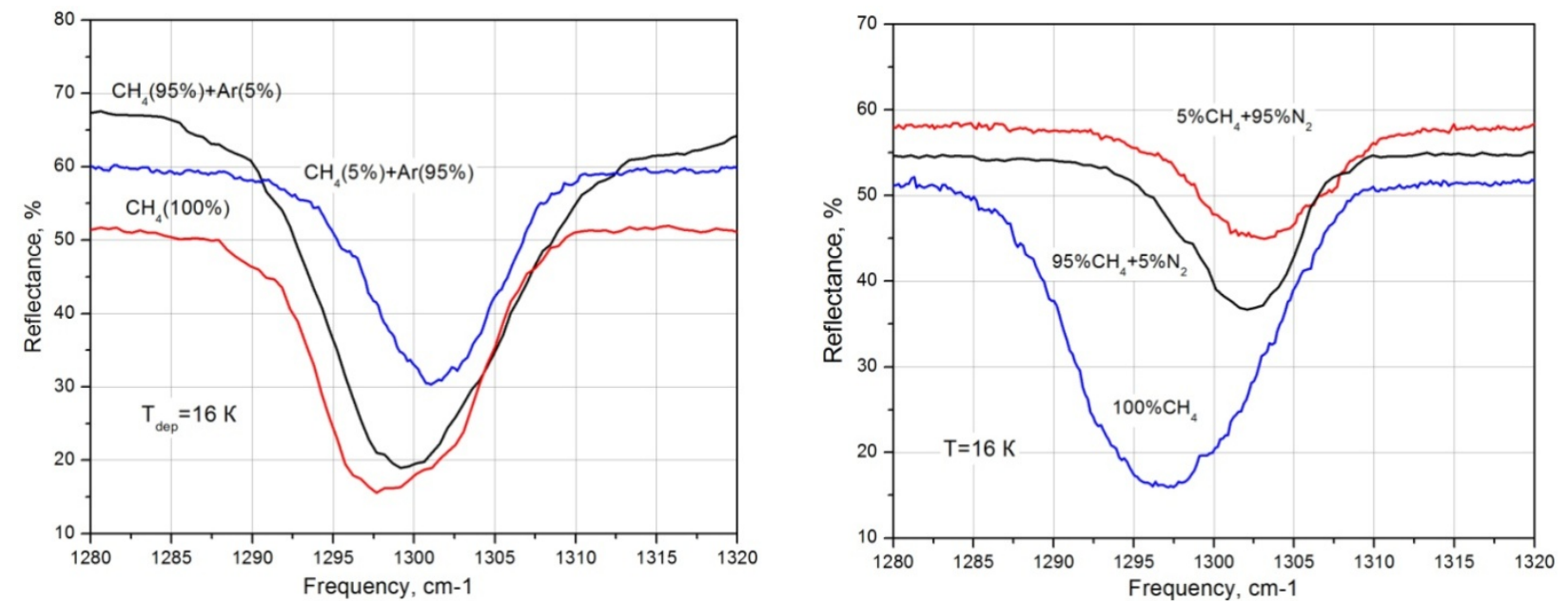

Figure 3 - IR spectra of cryocondensates of a mixture of methane with argon (left) and methane with nitrogen (right) at various concentrations. Condensation temperature $\mathrm{T}=16 \mathrm{~K}$

Figure 4 shows the spectra of cryofilms of $5 \%$ mixtures of methane with argon (left) and nitrogen (right) in the vicinity of the absorption band of deformation vibrations of methane at a condensation temperature $\mathrm{T}=16 \mathrm{~K}$ and subsequent heating of the films to $26 \mathrm{~K}$. As can be seen, passing through the temperature of the methane structural phase transition for a 5\% mixture (left) leads to a slight shift of the absorption bands to the long-wavelength part of the spectrum. At the same time, $95 \%$ of the mixture (right) was observed obvious broadening of the absorption band at $26 \mathrm{~K}$ as compared with the spectrum at $16 \mathrm{~K}$. It is obvious, that these changes are not significant as for pure methane (Figure 1). The offset of the position of the absorption maximum is of the order of $1 \mathrm{~cm}^{-1}$, while the absorption half-width has changed by approximately on $\Delta=5 \mathrm{~cm}^{-1}$. The magnitude of the absorption amplitude maintained practically unchanged.
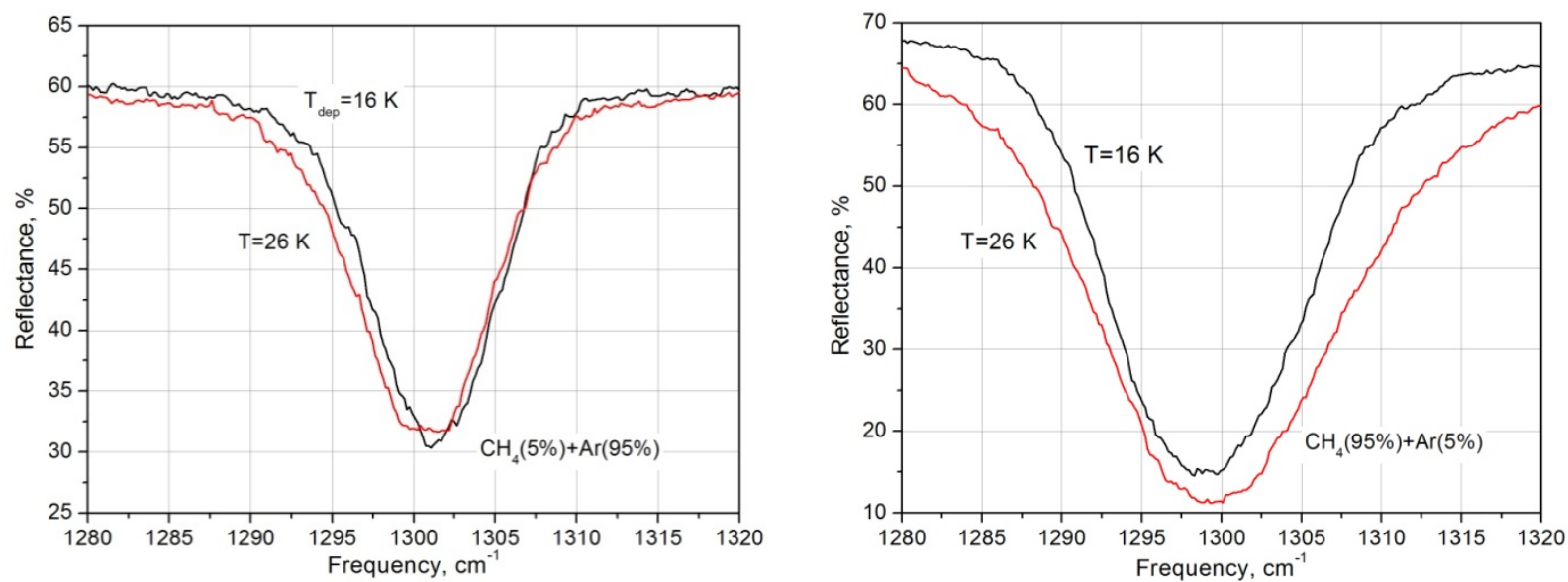

Figure 4 - IR spectra of cryocondensates of a mixture of 5\% methane $+95 \%$ argon (left) and $95 \%$ methane $+5 \%$ argon (right) at a condensation temperature $\mathrm{T}=16 \mathrm{~K}$ and heating $\mathrm{T}=26 \mathrm{~K}$

Similar data for mixtures of methane and nitrogen are shown in Figure 5. It can be seen that for the mixture with $5 \%$ of methane, the change in the position of the absorption band is insignificant and similar to the corresponding data for a mixture of methane and argon (left-hand inset in Figure 4). 
What cannot be said for the right-hand side of Figure 5. As can be seen, heating a methane film with 5\% nitrogen admixture results in both an insignificant broadening of the absorption band and a significant "red" shift of it from the value at the absorption minimum $v=1302 \mathrm{~cm}^{-1}$ at $16 \mathrm{~K}$ to $v=$ $1299 \mathrm{~cm}^{-1}$ at $26 \mathrm{~K}$. These changes in the position of the absorption band of a mixture of methane and nitrogen differ significantly from the data for a mixture of methane and argon (Figure 4).
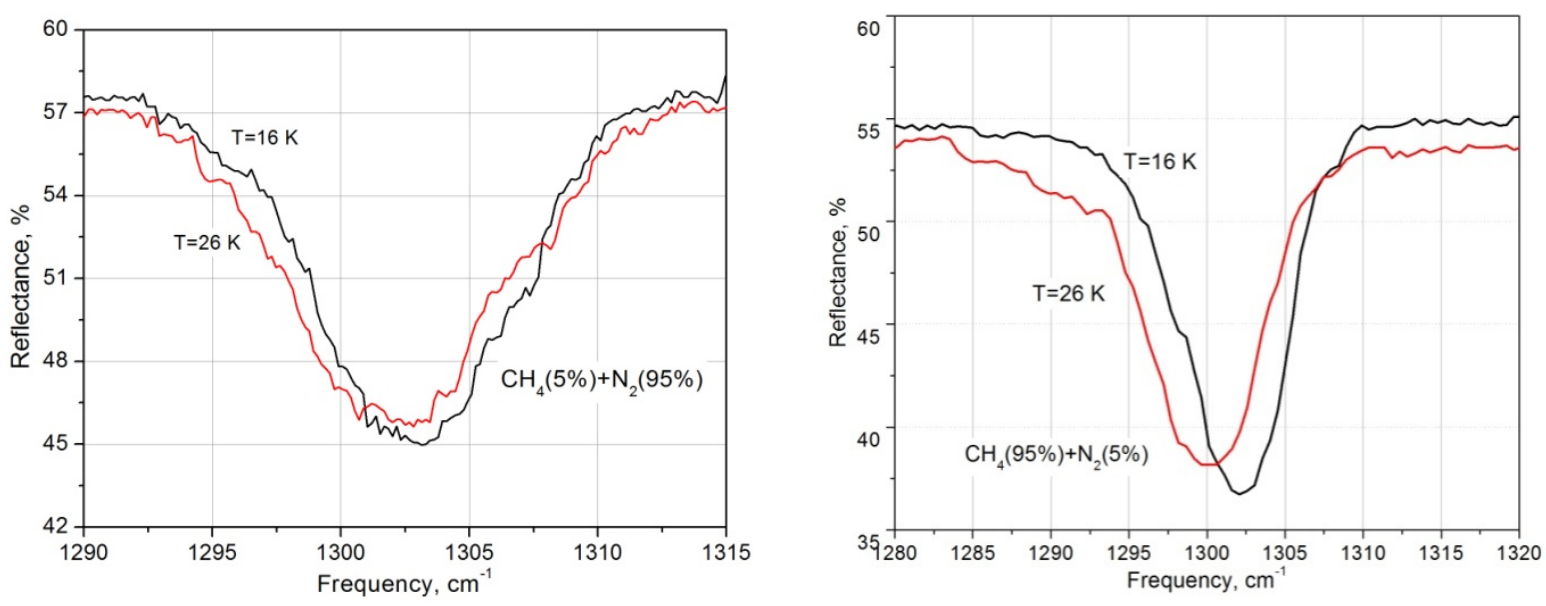

Figure 5 - IR spectra of cryocondensates of a mixture of 5\% methane $+95 \%$ nitrogen (left) and $95 \%$ methane $+5 \%$ nitrogen (right) at the condensation temperature $\mathrm{T}=16 \mathrm{~K}$ and heating $\mathrm{T}=25 \mathrm{~K}$

By analogy with the study of pure methane and other substances $[13,14]$ in this paper, we also use the method of obtaining thermograms of spectra transformation, recording changes in the signal of the IR spectrometer at a fixed frequency of observations. As a rule, a value is chosen at the half-width of the absorption of the characteristic vibration of the sample molecule, which is most sensitive to thermostimulated structural changes in the solid phase. In this paper, this method has demonstrated the reversibility of $\alpha-\beta$-transition cryodeposits of pure methane (Figure 2). The thermogram of the change in the absorption band $v_{4}$ position at the observation frequency $v=1295 \mathrm{~cm}^{-1}$ is presented below, in Figure 6. The data were obtained for a mixture of 95\% methane and 5\% argon.

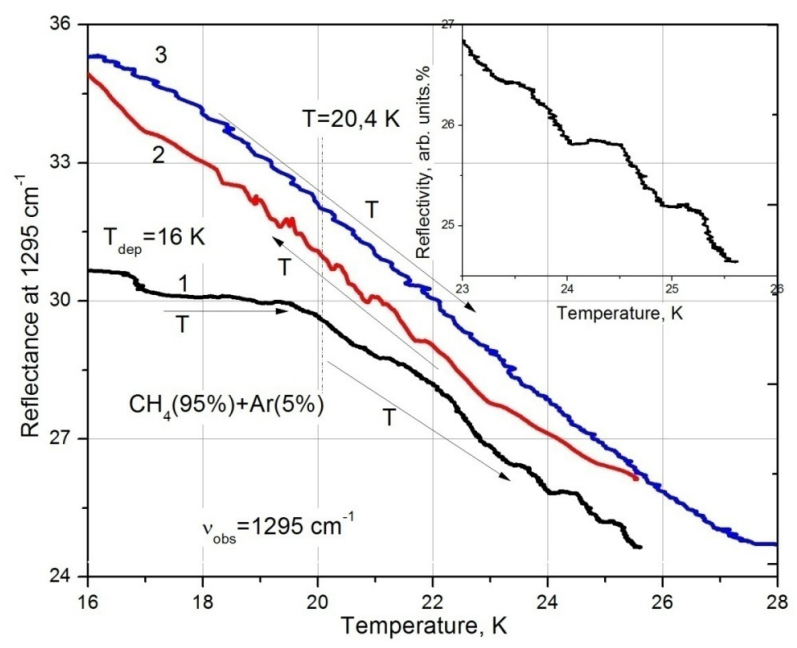

Figure 6 - The thermogram of the change in the position of the absorption band of the vibrations $v_{4}$ of the cryocondensates of mixture of $95 \%$ methane $+5 \%$ argon at the observation frequency $v=1295 \mathrm{~cm}^{-1}$ 
The sample was condensed at $16 \mathrm{~K}\left(T_{d e p}\right)$ after which it was heated to $26 \mathrm{~K}$ (the direction of the temperature change is indicated by arrows) with simultaneous measurement of the spectrometer signal at a frequency $v=1295 \mathrm{~cm}^{-1}$ (curve l). After, the sample was cooled down again to $16 \mathrm{~K}$ (curve 2) followed by heating up to its full evaporation (curve 3). In all cases, the sample passed the phase transition temperature $\mathrm{T}=20.4 \mathrm{~K}$ (in the figure, it is noted by the dotted line). To make a clear image, thermograms 1-3 are slightly shifted relatively to each other.

As clearly demonstrated by curve 1, there are structural transformation in the sample due to initial heating at around $20 \mathrm{~K}$, which are obviously related to $\alpha-\beta$-transition. This is indicated by a break in the thermogram at $20 \mathrm{~K}$ and a subsequent increase in the displacement velocity of the band $v_{4}$ with the temperature change. Subsequent increase in temperature (thermogram 2) leads to an increase the signal at the indicated frequency, which means a narrowing of the band $v_{4}$. However, it is important to note the fact that the character of thermogram 2 differs sharply from thermogram 1, which is expressed in the absence of a characteristic kink in the temperature region of the $\alpha-\beta$ transition. The thermogram 2 is a concave monotonic dependence of the IRS signal on the temperature decrease. At the same time, the subsequent growth of the temperature (thermogram 3) also demonstrates the monotonous but convex character of the change in the IRS signal with rising the temperature. Subsequent thermal cycling procedures 16-26-16 K indicate that thermograms obtained during thermal cycling are in good agreement with thermograms 2 and 3 , respectively. That is, thermograms 2 and 3 are internally interconnected and we believe that the core of this interrelation is the spin-nuclear conversion processes of transition to equilibrium spin states [15-20] whose velocity, in our opinion, depends not only on temperature but also on the direction of its changes. The inset in the right-hand corner of the figure demonstrates an enlarged fragment of thermogram 1, showing the step-by-step character of the signal change in the spectrometer. Similar behavior of cryocondensates of pure methane in the process of cryodeposition was previously described in our work [14].

\section{Conclusions}

The experimental data obtained for the thermally stimulated transformations in pure methane cryofilms demonstrate the relationship between the shift of the absorption band $v_{4}$ and the change in the film temperature. This is due to the spin-nuclear conversion processes occurring in the samples of methane cryocondensates, which is superimposed on the actual $\alpha-\beta$ phase transition. In addition, each equilibrium value corresponds to the equilibrium concentration ratio of the spin-nuclear modifications of methane (meta-ortho-para) and the corresponding position of the absorption band. However, as the conversion rate is the function of the temperature, the time for achieving this equilibrium value depends on the direction of temperature change.

The obtained vibrational spectra of methane cryodeposits mixed with argon and nitrogen in various ratios demonstrate the relationship between the concentrations of nitrogen or argon and the position of the absorption band $v_{4}$ - lower methane concentration leads to greater band offset $v_{4}$ to the "blue" part of the spectrum. Heating of the mixture of methane and argon from the deposition temperature $(T=16 \mathrm{~K})$ to $\mathrm{T}=26 \mathrm{~K}$ leads to broadening of the spectrum. At the same time for the mixture of methane and nitrogen, heating of the mixture of methane with 5\% nitrogen leads not only to slight broadening of the absorption band, but also to its substantial "red" displacement.

Both 5\% mixtures of argon and nitrogen in methane exhibit violation of reversibility of methane's $\alpha-\beta$ transition. Whereas initial annealing from $16 \mathrm{~K}$ to $26 \mathrm{~K}$ demonstrates transition from the $\beta$-phase to the $\alpha$-phase at around $20 \mathrm{~K}$, the subsequent temperature rises and thermocycling in the same temperature range result in a change in trajectory of the spectrometer signal, with no obvious relationship with the initial heating curve. The thermograms of heating and cooling are internally interconnected through the process of transition to the equilibrium of spin states of methane. The similarity of hysteresis between the heating and cooling thermograms can be observed due to the dynamics of attaining equilibrium spin states depending not only on temperature, but also on the direction of its change. 


\section{References}

1. A. J. Nijman, A. J. Berlinsky. Theory of nuclear spin conversion in the $\beta$ phase of solid methane // Phys. Rev. Lett. - 1977. - Vol. 38. - P. 408.

2. A. J. Nijman, A. J. Berlinsky. Theory of nuclear spin conversion in the $\beta$ phase of solid $\mathrm{CH}_{4} / /$ Canadian. Jour. of Phys. - 1980. - Vol. 58. - P. 1049.

3. Yi. Pon-Nyong, I. Ozier, C.H. Anderson. Theory of nuclear hyperfine interactions in spherical-top molecules // Phys. Rev. - 1968. - Vol. 165. - P. 92.

4. P. Cacciani, J. Cosléou, M. Khelkhal, P. Čermák, C. Puzzarini. Nuclear spin conversion in $\mathrm{CH}_{4}$ : A multichannel relaxation mechanism // J Phys Chem A. -2016. - Vol. 120. - P. 173-182.

5. J. T. Hougen, T. Oka. Nuclear spin conversion in molecules // Science. - 2005. - Vol. 310. - No. 5756. - P. 1913-1914.

6. F.H. Frayer, G. E. Ewing. Nuclear-spin conversion and vibration-rotation spectra of methane in solid argon // Journal of Chemical Physics. - 1968. - Vol. 48. - P. 781.

7. P. L. Chapovsky. Nuclear spin conversion in formaldehyde // J. of Molecular Structure. - 2001. - Vol. 599. No. 1-3. - P. 337-345.

8. Y. Miyamoto, M. Fushitani, D. Ando, T. Momose. Nuclear spin conversion of methane in solid parahydrogen // J. of Chem. Phys. - 2008. - Vol. 128. - P. 114502.

9. G. Herzberg. Infrared and Raman spectra of polyatomic molecules. - New York, 1945. -647 p.

10. F. H. Frayer, G. E. Ewing. Spectroscopic observation of nuclear spin conversion in methane // J. Chem. Phys. 1967. - Vol. 46. - P. 1994-1995

11. A. Drobyshev, N. Atapina, D. Garipogly. IR-spectrum and structure of water-vapor cryocondensates // Fizika Nizkikh Temperatur. - 1993. - Vol. 19 (5). - P. 567.

12. A. Aldiyarov, M. Aryutkina, A. Drobyshev, V. Kurnosov. IR spectroscopy of ethanol in nitrogen cryomatrices with different concentration ratios // LTP. - 2011. - Vol. 37 (6). - P. 524.

13. A. Drobyshev, A. Aldiyarov, E. Korshikov, V. Kurnosov, D. Sokolov, N. Tokmoldin. Structure and phase transition peculiarities in solid nitrous oxide and attempts at their explanation // LTP. - 2013. - Vol. 39 (5). - P. 460.

14. A. Drobyshev, A. Aldiyarov, D. Sokolov, A. Shinbayeva. IR Studies of the Spin-Nuclear Conversion in the vicinity of $\alpha$ - $\beta$-transition in cryodeposited methane films // J. LTP. - 2017. - Vol. 187 (5-6). - P. 742.

15. I.Ya. Minchina, V.G. Manzhelii, M.I. Bagatskii, O.V. Sklyar, D.A. Mashchenko, M.A. Pokhodenko. Heat capacity of methane-krypton solid solutions. Conversion effect // Fizika Nizkikh Temperatur. - 2011. - Vol. 27 (7). - P. 773.

16. E. Pisarska, P. Stachowiak, A. Jezowski. Observation of relaxation of molecular spins in $\mathrm{CH}_{4}$ and $\mathrm{CD}_{4}$ crystals in thermal conductivity experiment // Fizika Nizkikh Temperatur. - 2007. - Vol. 33(6/7). - P. 768.

17. M. Miki, T. Momose. Rovibrational transition and nuclear spin conversion of methane in parahydrogen crystals // Fizika Nizkikh Temperatur. - 2000. - Vol. 26. - No. 9/10. - P. 899-908.

18. H. P. Hopkins Jr., P. L. Donoho, K. S. Pitzer. Oxygen catalysis of nuclear spin species conversion in solid methane // The Journal of Chemical Physics. - 1967. - Vol. 47. - P. 864-865.

19. B.Ya. Gorodilov, A.I. Krivchikov, O.A. Korolyuk. Effekt spin-yadernoy konversii v teploprovodnosti metana // Fizika Nizkikh Temperatur. - 2005. - Vol. 31. - No. 10. - P. 1158-1162. In Russian.

20. T.V. Lokotosh. Spinovaya konversiya v $\beta$-phaze metana // Fizika Nizkikh Temperatur. - 1988. - Vol. 14. No.5. - P. 198-209. In Russian. 